\title{
Verificação de modelos computacionais para placas com enrijecedores submetidas a carregamento transversal uniforme
}

\section{Verification of computational models for stiffened plates under uniform transverse loading}

Grégori da Silva Troina1, João Pedro Teixeira Peixoto de Queiroz ${ }^{2}$, Marcelo Langhinrichs Cunha ${ }^{3}$, Luiz Alberto Oliveira Rocha ${ }^{4}$, Elizaldo Domingues dos Santos ${ }^{5}$, Liércio André Isoldi ${ }^{6}$

\section{RESUMO}

Este artigo apresenta a verificação de modelos computacionais para a análise da deflexão em placas reforçadas com enrijecedores. No presente estudo, essas placas enrijecidas são analisadas quando submetidas a esforços transversais uniformemente distribuídos. A verificação é realizada por meio da comparação entre os resultados numéricos obtidos neste estudo com resultados encontrados em referências científicas publicadas por outros autores. Os modelos computacionais foram desenvolvidos no software ANSYS, que é baseado no Método dos Elementos Finitos (MEF), e utilizam dois tipos de elementos finitos diferentes: SHELL93 e SOLID95. São utilizadas duas variações na geometria de cada um desses dois tipos de elementos, totalizando, portanto, quatro modelos computacionais distintos entre si. Os testes de independência de malha evidenciam um comportamento convergente das simulações realizadas para os dois exemplos de placa retangular analisados: uma com apenas um enrijecedor central e outra com dois enrijecedores ortogonais em formato de cruz. Os resultados da verificação dos modelos mostram que os quatro tipos de elementos apresentam boa concordância entre si em todos os problemas avaliados. Além disso, os resultados gerados pelos modelos computacionais são compatíveis com as referências científicas utilizadas para comparação.

Palavras-chave: Deflexão. Placas Enrijecidas. Simulação Numérica. Verificação.

\section{ABSTRACT}

This paper presents the verification of computational models in the analysis of stiffened plates under deflection. In this study, these stiffened plates are analyzed for the situation where there is a transversal loading uniformly distributed over their surface. The verification is based on comparison among the numerical results obtained by this study and results found on scientific publications of distinct authors. The computational models were developed on the software ANSYS, which is based on the Finite Elements Method (FEM), and utilizes two different elements: SHELL93 and SOLID95. In order to accomplish this work, two distinguished geometries are defined for each of the elements under consideration, resulting in four different computational models. The grid independence tests show that the results from the simulations present a converging pattern for both rectangular plates examples: one with only one central stiffener and other with two orthogonal stiffeners. At the same time, the product of the verification of the models shows that the four types of elements present an agreement in their outcomes for all the analysis covered by this work. Besides, the results of the computational models are in accordance with those obtained from the scientific literature.

Keywords: Deflection. Stiffened Plates. Numerical Simulation. Verification.
${ }^{1}$ Mestre em Engenharia Oceânica. Programa de Pós-Graduação em

Engenharia Oceânica (PPGEO) da Universidade Federal do Rio

Grande (FURG) - Rio Grande/RS.

E-mail: gregori.troina@gmail.com

${ }^{2}$ Engenheiro Mecânico Naval. Escola de Engenharia (EE) da FURG - Rio Grande/RS.

${ }^{3}$ Graduando em Engenharia Mecânica Empresarial. EE da FURG - Rio Grande/RS.

${ }^{4}$ Doutor em Engenharia

Mecânica. Professor do Programa de Pós-Graduação em Engenharia Mecânica da Universidade do Vale do Rio dos Sinos (Unisinos) São Leopoldo/RS.

${ }^{5}$ Doutor em Engenharia Mecânica. Professor do PPGEO da EE da FURG - Rio Grande/RS.

${ }^{6}$ Doutor em Engenharia Mecânica. Professor do PPGEO da EE da FURG - Rio Grande/RS. 


\section{INTRODUÇAOO}

Estruturas que utilizam placas com enrijecedores são amplamente empregadas na indústria aeronáutica e na construção civil, além de assumirem um papel importante no setor de construção e reparação naval. Devido à complexidade dessas estruturas, métodos analíticos para determinação dos seus estados de tensão e campos de deslocamentos são raros. Além disso, considerando os altos custos de equipamento e material inerentes aos ensaios experimentais, os profissionais de engenharia estrutural têm recorrido, frequentemente, à modelagem computacional para estudar esses problemas de engenharia.

Entre as pesquisas existentes neste tema, é possível destacar a de Rossow e Ibrahimkhail (1978) que aplicaram o Método das Restrições em análises estáticas, via elementos finitos, de placas enrijecidas com reforços concêntricos e excêntricos. Foram solucionados numericamente exemplos de placa quadrada com apenas um enrijecedor e placa retangular com dois enrijecedores se cruzando ortogonalmente. Nessas análises foram utilizados 2 elementos triangulares para modelar 1/4 da placa e foram empregados polinômios de aproximação de ordem 4 até ordem 9. Além disso, para obter uma checagem independente das soluções, esses problemas foram resolvidos com os softwares NASTRAN e STRUDL.

Já Bedair (1997) analisou placas enrijecidas sob carregamento transversal utilizando o método da Programação Sequencial Quadrática. Nesse estudo a estrutura foi idealizada como uma placa conectada de forma rígida a vigas enrijecedoras. A energia de deformação dessa estrutura idealizada foi obtida em termos de funções generalizadas de deslocamento dentro e fora do plano, onde os coeficientes dessas funções funcionavam como variáveis de projeto. A programação sequencial quadrática foi então utilizada para determinar a magnitude desses coeficientes a fim de minimizar o potencial total de energia do sistema.

Placas reforçadas por vigas também foram abordadas no artigo publicado por Sapountzakis e Katsikadelis (2000). Nesse trabalho os autores aplicaram uma metodologia que considerava os esforços e deformações no plano da placa bem como os esforços e deformações axiais nas vigas, sendo esses esforços avaliados nas regiões da interface de união entre os enrijecedores e a placa. Isso permitiu o cálculo dos esforços de cisalhamento nessas regiões de ligação entre a placa e os reforços, parâmetro importante para o projeto de placas reforçadas pré-fabricadas ou executadas em material 
compósito. A solução das equações diferenciais do problema foi obtida através do Método da Equação Análoga e um dos exemplos analisados para verificar a eficácia do método foi uma placa retangular simplesmente apoiada com um enrijecedor central também de seção transversal retangular com diferentes alturas.

Em Silva (2010) foram realizados estudos numéricos em lajes nervuradas, tratando essas estruturas como um sistema placa-vigas e mostrando que a consideração da excentricidade entre placa e viga de reforço resultava em redução dos deslocamentos. Os pavimentos foram simulados com o software ANSYS utilizando elementos de casca (SHELL63) para modelar a placa e elementos de viga (BEAM44) para modelar os enrijecedores. Os exemplos analisados foram, entre outros, os mesmos apresentados por Rossow e Ibrahimkhail (1978), Bedair (1997) e Sapountzakis e Katsikadelis (2000).

O presente artigo utiliza o software ANSYS para verificar modelos computacionais de placas enrijecidas por meio da simulação de dois exemplos numéricos dentre os estudados por Rossow e Ibrahimkhail (1978), Bedair (1997), Sapountzakis e Katsikadelis (2000) e Silva (2010). Os modelos computacionais foram construídos com base no Método dos Elementos Finitos (MEF), sendo utilizados, para isso, o tipo de elemento SHELL93 nas formas triangular e quadrilátera e o tipo SOLID95 nas versões espaciais tetraédrica e hexaédrica.

\section{MATERIAIS E METODOS}

A seguir, serão descritos os principais materiais e métodos considerados na realização desse trabalho.

\subsection{Placas Enrijecidas}

Segundo Szilard (2004), placas são componentes estruturais planos que possuem uma de suas dimensões, referida como espessura, muito menor quando comparada às outras. Elementos estruturais formados por placas podem ser encontrados em diferentes áreas da engenharia, como nas indústrias automobilística, aeronáutica, de construção civil e de construção e reparação naval. As placas enrijecidas, por sua vez, são obtidas pela fixação de vigas esbeltas à superfície inferior ou superior de uma placa base, com o objetivo de aumentar a rigidez da nova estrutura formada, podendo, por exemplo, ser formadas pela soldagem de perfis dispostos longitudinalmente e/ou transversalmente. 
Essa prática confere ao conjunto uma melhor capacidade para resistir às combinações de esforços de flexão, torção, entre outros carregamentos.

Salomon (2000) divide as diferentes abordagens disponíveis para a análise de placas enrijecidas em três grandes categorias: modelo de grelhas, modelo de placa ortotrópica e sistemas de placa-vigas.

De acordo com as pesquisas realizadas por Salomon (2000), a idealização de uma placa enrijecida como uma grelha requer que a placa possua uma largura efetiva variando em torno de $50 \%$ a $80 \%$ do espaçamento entre os reforços. Este método de análise fornece deflexões com erros normalmente de até $5 \%$ e, ocasionalmente, de até 10\%, enquanto as tensões diferem geralmente entre $10 \%$ a $20 \%$ dos valores experimentais.

Segundo Szilard (2004), embora o real comportamento estrutural de placas reforçadas com enrijecedores não possa ser completamente substituído pelo de placas ortotrópicas, dados experimentais indicam boa concordância de resultados ao aplicar tal idealização, desde que os reforços sejam relativamente pequenos, estejam próximos e uniformemente espaçados. A ideia básica desse método é a transformação de uma placa enrijecida em uma placa ortotrópica equivalente de espessura única, sem a presença de enrijecedores.

Já as análises que tratam placas enrijecidas como um sistema composto por placa e vigas de reforço refletem o comportamento real ou mais próximo do comportamento verdadeiro do problema físico. Devido às dificuldades em resolver esses tipos de problemas de forma analítica, essa metodologia só foi impulsionada com o advento dos computadores digitais, que possibilitaram a solução desses modelos através de métodos numéricos. Entre os métodos numéricos, o Método dos Elementos Finitos (MEF) é o mais poderoso e, de fato, o esforço de pesquisa é no sentido de tentar conseguir modelos de elementos finitos precisos e eficientes para descrever de maneira apropriada o comportamento mecânico de placas reforçadas com enrijecedores (SALOMON, 2000).

\subsection{Método dos Elementos Finitos (MEF)}

Zienkiewicz (1971) e Bathe (1996) resumem a análise por elementos finitos em quatro etapas: criação da geometria (domínio computacional), geração da malha (discretização do domínio computacional em partes elementares), aplicação das condições de contorno e carregamento externo e, por fim, a solução do problema. 
$\mathrm{Na}$ análise de estruturas de comportamento linear elástico, via elementos finitos, a formulação matemática é derivada da aplicação do princípio dos trabalhos virtuais. No caso específico da análise estática, o objetivo é quantificar a magnitude dos esforços internos e dos deslocamentos que se manifestam em qualquer sistema estrutural quando o mesmo é submetido a um carregamento arbitrário, desprezando-se o efeito das forças de amortecimento (velocidades e atritos internos) e das forças de inércia (massa e acelerações). Os deslocamentos desconhecidos são obtidos através da solução de um sistema de equações algébricas que pode ser representado por (MARINHO, 2002):

$$
[K] \cdot\{u\}=\{F\}
$$

onde $\{u\}$ é o vetor dos deslocamentos nodais desconhecidos, $\{F\}$ é o vetor de carga externa e $[K]$ é a matriz de rigidez global da estrutura, a qual é obtida por meio das relações deformação-deslocamento, tensão-deformação e propriedades do material, considerando a estrutura após a malha de elementos finitos já ter sido gerada.

\subsection{Software ANSYS}

A análise estrutural é uma das aplicações mais comuns do MEF. Dentro desse contexto, existem vários programas computacionais com a proposta de utilizar esse método numérico para solucionar uma ampla gama de problemas complexos relacionados às estruturas. Nas análises realizadas neste trabalho, é utilizado o software ANSYS $^{\circledR}$ Mechanical APDL, que é desenvolvido por ANSYS ${ }^{\circledR}$ Inc., uma companhia especializada em programas de simulação numérica multidisciplinar para engenharia. As análises estruturais disponíveis neste programa incluem: estática (linear e não-linear), modal, espectral, dinâmica e de flambagem. Em todas essas análises, as variáveis primárias calculadas são os deslocamentos nodais, enquanto que as demais grandezas como deformações, tensões e forças de reação são derivadas dos valores de deslocamentos previamente obtidos para todo o domínio computacional (ANSYS, 2009).

\subsection{Tipos de Elementos Finitos Utilizados}

O elemento SHELL93 foi um dos tipos de elementos utilizados nesse trabalho, justamente por ser adequado para modelar problemas de cascas envolvendo estruturas planas ou curvas de pequena espessura. Mais especificamente, esse elemento possui 6 graus de liberdade em cada um dos seus 8 nós: translação nas direções nodais $x, y$, e $z$ e 
rotação em torno dos eixos nodais $x, y$, e $z$. As funções de interpolação utilizadas na sua formulação matemática são do tipo quadrática. Dentre as suas capacidades de análise estão: plasticidade, grandes deslocamentos e grandes deformações. Destaca-se como considerações estruturais importantes o fato da tensão na direção normal ao plano do elemento variar linearmente ao longo da espessura e a tensão de cisalhamento transversal ser assumida como constante ao longo da espessura. Além disso, esse elemento possui uma versão simplificada para geração de malhas com formato triangular como mostrado na Fig. 1a (ANSYS, 2009).

Já o SOLID95, que é o outro tipo de elemento utilizado nesse artigo, trata-se de um elemento de alta ordem que pode tolerar formas irregulares sem perda significativa na acurácia dos resultados. Também é adequado para modelar geometrias curvas e, como mostrado na Fig.1b, possui as variações geométricas espaciais: tetraédrica, piramidal e prismática. Esse elemento é definido por 20 nós com 3 graus de liberdade cada: translações nas direções nodais $x, y$ e $z$, sendo capaz de analisar plasticidade, fluência, grandes deslocamentos e grandes deformações (ANSYS, 2009).

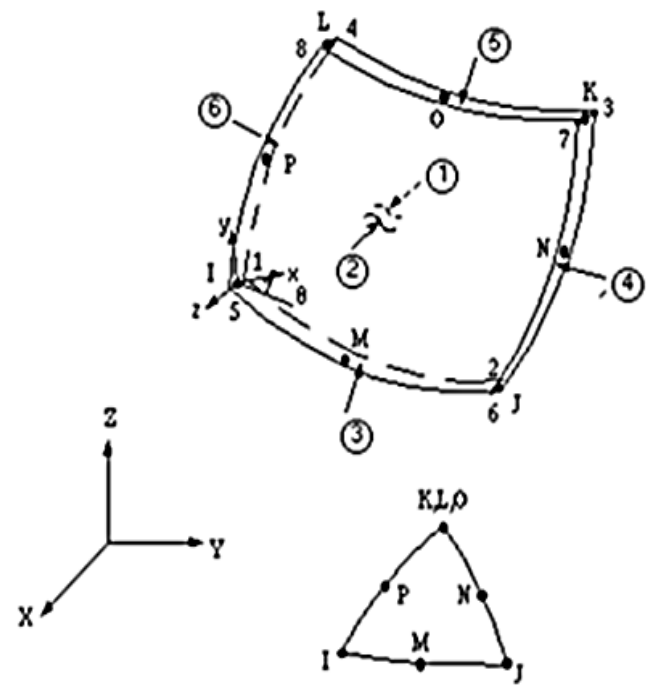

a)
Opção Triangular

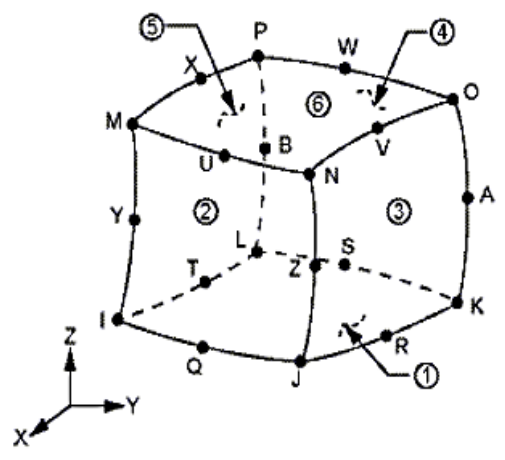

b)

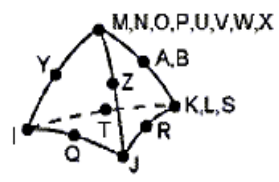

Opção Tetraédrica
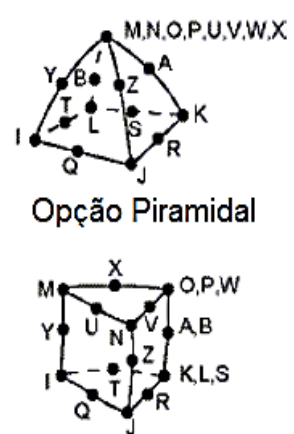

Opção Prismática

Figura 1. a) Elemento tipo SHELL93 e b) Elemento tipo SOLID95.

(FONTE: Adaptada de ANSYS, 2009)

\section{RESULTADOS E DISCUSSÖES}

\subsection{Placa Retangular Simplesmente Apoiada com um Enrijecedor Central}

Uma placa retangular com um enrijecedor central no sentido longitudinal submetida a uma carga transversal uniformemente distribuída de $10 \mathrm{kN} / \mathrm{m}^{2}$ e condições de apoio 
simples em todas as bordas, como mostra a Fig. 2, foi considerada. A análise se deu pela determinação da deflexão no centro da placa, considerando dois valores de altura de enrijecedor $h_{s}=2,00 \mathrm{~m}$ e $h_{s}=1,25 \mathrm{~m}$. O módulo de elasticidade e coeficiente de Poisson do material utilizados foram, respectivamente, $30 \mathrm{GPa}$ e 0,154 . O mesmo problema fora estudado por Sapountzakis e Katsikadelis (2000) e por Silva (2010).

A Figura 3 mostra as malhas M1 para exemplificar a discretização do domínio computacional da placa com um enrijecedor de 2,00 $\mathrm{m}$ de altura, para os quatro modelos computacionais propostos no presente estudo.

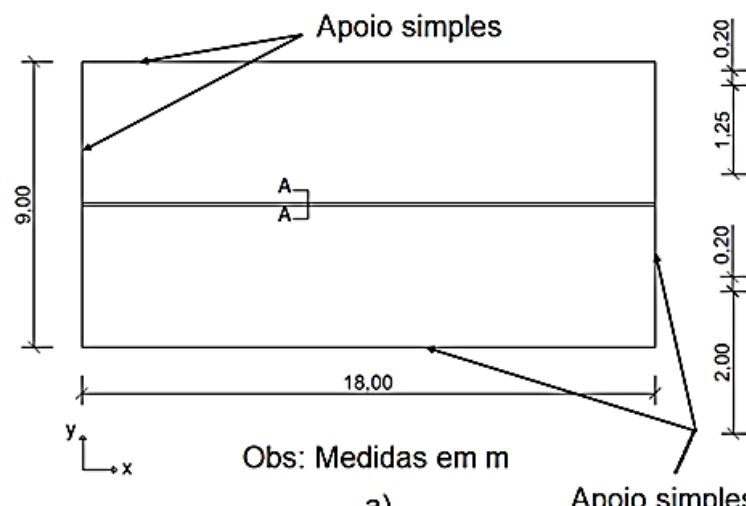

a)

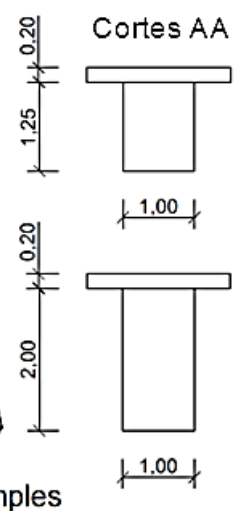

$\nmid 1.00 \nmid$

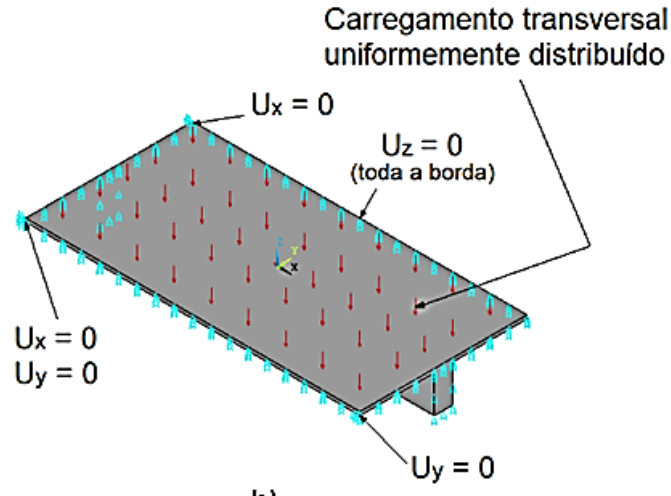

b)

Figura 2. Placa retangular com um enrijecedor central: (a) modelo físico [Adaptada de Silva (2010)] e (b) condições de contorno e carregamento.

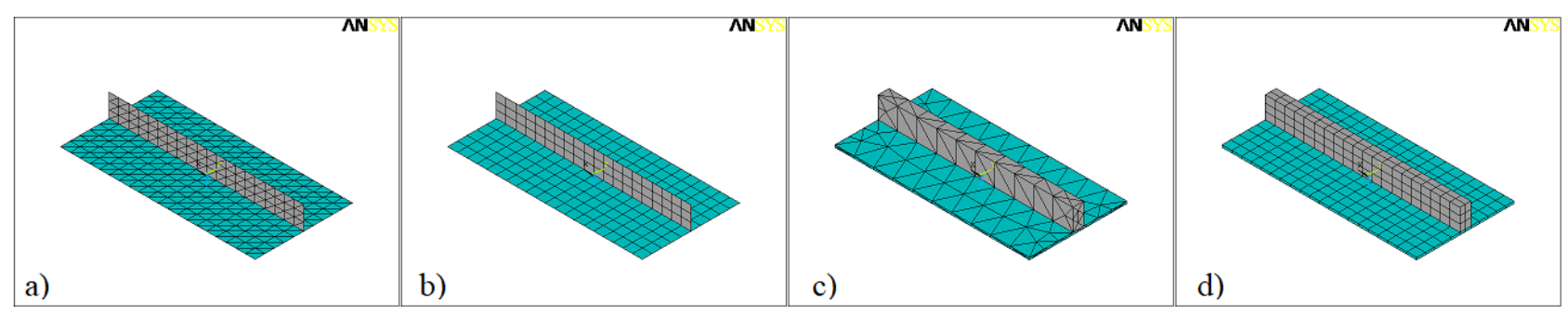

Figura 3. Malhas M1 para o domínio computacional da placa retangular com um enrijecedor central $\left(h_{s}=2,00 \mathrm{~m}\right)$ : a) SHELL93 - Triangular, b) SHELL93 - Quadrilátero, c) SOLID95 - Tetraédrico e d) SOLID95 - Hexaédrico.

Além das malhas M1 mostradas na Fig. 3, outros cinco refinamentos de malha (M2, M3, M4, M5 e M6) foram gerados para cada modelo computacional, cujos resultados de deslocamento transversal no ponto central da placa com $h_{s}=2,00 \mathrm{~m}$ estão apresentados 
no gráfico da Fig. 4. Já no gráfico da Fig. 5, são mostrados os resultados obtidos para uma análise similar de uma placa com $h_{s}=1,25 \mathrm{~m}$.

A malha considerada independente para o caso com $h_{s}=2,00 \mathrm{~m}$ (Fig. 4) apresentou uma diferença percentual em relação à malha seguinte de até $0,06 \%$. Para o caso com $h_{s}=1,25 \mathrm{~m}$ (Fig. 5), a diferença entre a malha independente e a malha seguinte foi de até $0,02 \%$.

Analisando as Figs. 4 e 5 é possível observar que todos os modelos numéricos propostos neste trabalho convergem em torno de um valor específico e estão em concordância com os resultados apresentados por Silva (2010).

Os resultados obtidos para a deflexão do ponto central da placa em cada um dos modelos computacionais, com sua respectiva malha independente, são comparados na Tabela 1 com os resultados publicados por Sapountzakis e Katsikadelis (2000) e por Silva (2010).

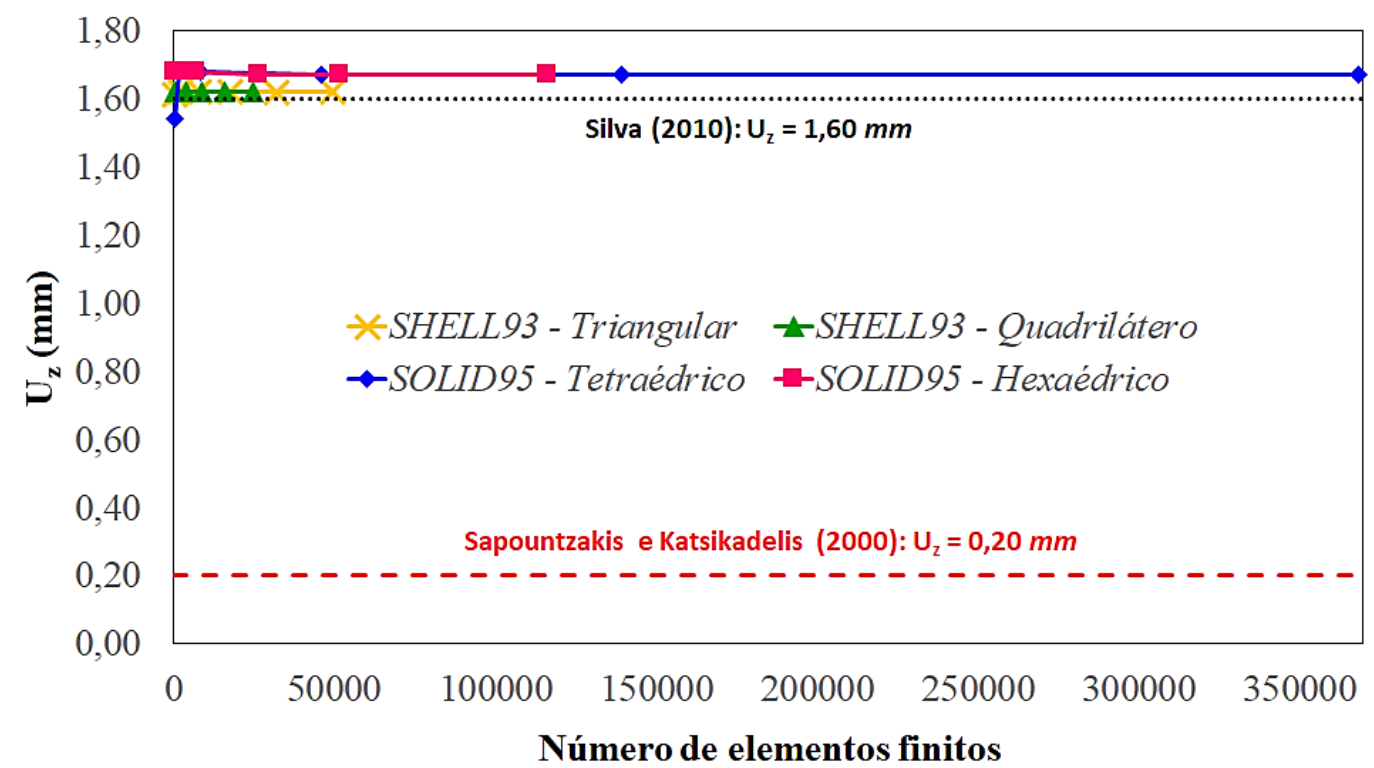

Figura 4. Resultados numéricos e análise de convergência de malha: $h_{s}=2,00 \mathrm{~m}$. 


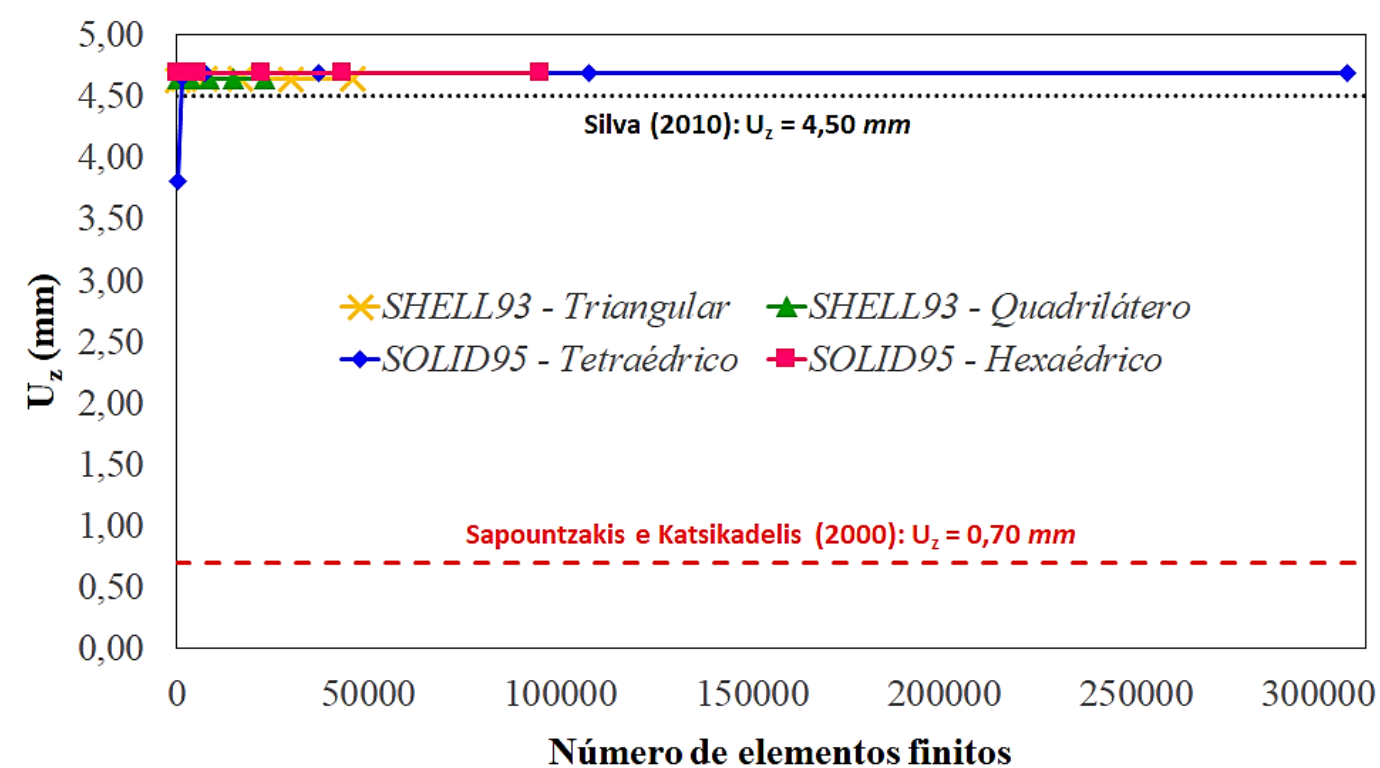

Figura 5. Resultados numéricos e análise de convergência de malha: $h_{s}=1,25 \mathrm{~m}$.

Tabela 1. Resultados para enrijecedor de altura $h_{s}=2,00 \mathrm{~m}$ e $h_{s}=1,25 \mathrm{~m}$.

\begin{tabular}{|c|c|c|c|c|c|c|c|}
\hline \multirow{2}{*}{\multicolumn{2}{|c|}{$\begin{array}{l}\text { Altura do } \\
\text { Enrijecedor } h_{s} \\
(\mathrm{~m})\end{array}$}} & \multicolumn{2}{|c|}{ SHELL 93} & \multicolumn{2}{|c|}{ SOLID 95} & \multirow{2}{*}{$\begin{array}{l}\text { Silva } \\
(2010)\end{array}$} & \multirow{2}{*}{$\begin{array}{c}\text { Sapountzakis } \\
\text { e Katsikadelis } \\
\text { (2000) }\end{array}$} \\
\hline & & Triangular & Quadrilátero & Tetraédrico & Hexaédrico & & \\
\hline \multirow{2}{*}{2,00} & Malha & $\begin{array}{l}\text { M1: } 520 \\
\text { elementos }\end{array}$ & $\begin{array}{l}\text { M1: } 260 \\
\text { elementos }\end{array}$ & $\begin{array}{l}\text { M4: } 46.005 \\
\text { elementos }\end{array}$ & $\begin{array}{l}\text { M3: } 6.880 \\
\text { elementos }\end{array}$ & ---- & ---- \\
\hline & $U_{z}(\mathrm{~mm})$ & 1,61 & 1,61 & 1,67 & 1,67 & 1,60 & 0,20 \\
\hline \multirow{2}{*}{1,25} & Malha & $\begin{array}{l}\text { M2: } 1.840 \\
\text { elementos }\end{array}$ & $\begin{array}{l}\text { M1: } 240 \\
\text { elementos }\end{array}$ & $\begin{array}{l}\text { M4: } 37.183 \\
\text { elementos }\end{array}$ & $\begin{array}{l}\text { M3: } 5.680 \\
\text { elementos }\end{array}$ & ---- & ---- \\
\hline & $U_{z}(\mathrm{~mm})$ & 4,64 & 4,64 & 4,69 & 4,69 & 4,50 & 0,70 \\
\hline
\end{tabular}

$\mathrm{Na}$ Tabela 1, observa-se boa concordância entre os resultados deste trabalho com os resultados obtidos por Silva (2010), que também resolve pelo Método dos Elementos Finitos, enquanto que o método utilizado por Sapountzakis e Katsikadelis (2000) produz resultados bastante diferentes. Cabe destacar que o modelo numérico empregando o elemento finito tridimensional SOLID95 Hexaédrico, pela sua quantidade de nós e regularidade da malha, é o modelo que produz resultados com maior acurácia, uma vez que para esse elemento não há simplificações na teoria da elasticidade como as que são feitas para os elementos do tipo casca. 
A Figura 6 mostra a configuração deformada da placa enrijecida para o caso com $h_{s}=2,00 \mathrm{~m}$, com o valor do deslocamento transversal no centro da placa obtido em cada modelo computacional com sua respectiva malha independente (ver Fig. 4 e Tabela 1).

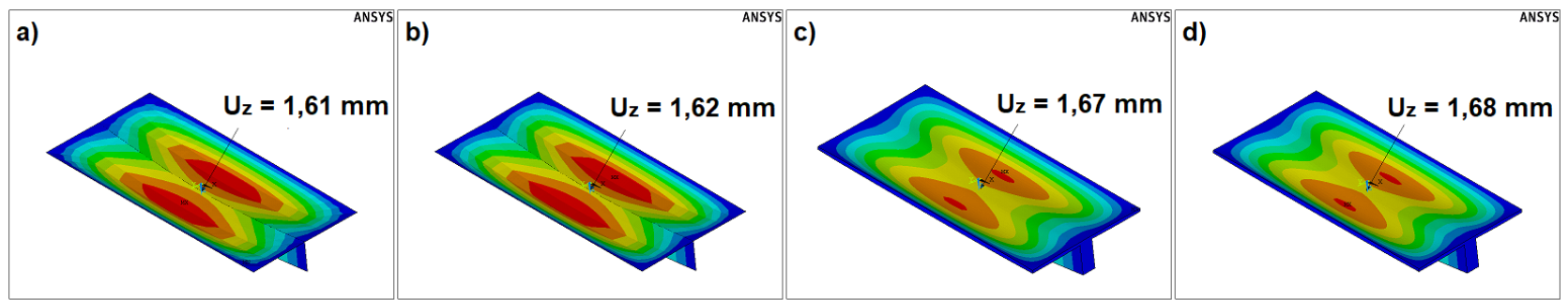

$0 \mathrm{~mm}$

$2,16 \mathrm{~mm}$

Figura 6. Deformada da placa com um enrijecedor central $\left(h_{s}=2,00 \mathrm{~m}\right)$ : a) SHELL93 Triangular, b) SHELL93 - Quadrilátero, c) SOLID95 - Tetraédrico e d) SOLID95 Hexaédrico.

Portanto, considerando os resultados apresentados nas Figs. 4 e 5 e na Tabela 1, junto com as configurações deformadas mostradas na Fig. 6, pode-se dizer que os quatro modelos numéricos propostos, para esse tipo de estrutura com apenas um enrijecedor, foram devidamente verificados.

\subsection{Placa Retangular Simplesmente Apoiada com dois Enrijecedores Ortogonais}

Nesse exemplo, uma placa retangular, com dois enrijecedores ortogonais que se cruzam exatamente na região central da placa, foi submetida a uma carga transversal uniformemente distribuída de $0,006895 \mathrm{kN} / \mathrm{cm}^{2}$ e condições de apoio simples em todas as suas bordas, incluindo as bordas dos enrijecedores, como mostra a Fig. 7. A análise se deu também pela determinação da deflexão transversal no centro da placa. O módulo de elasticidade e coeficiente de Poisson do material utilizados nessa modelagem foram, respectivamente, 206,85 GPa e 0,3. Esse mesmo problema já fora estudado por Rossow e Ibrahimkhail (1978), Bedair (1997) e Silva (2010).

Analogamente ao caso anterior, os valores de deflexão no ponto central da placa obtidos com os 4 modelos computacionais propostos no presente trabalho são apresentados em um gráfico de dispersão de pontos (Fig. 8) para seis refinamentos de 
malha diferentes, sendo considerada a malha independente para esse caso aquela que apresentou diferença percentual relativa à malha seguinte de até 0,09\%, considerando todos os 4 modelos.

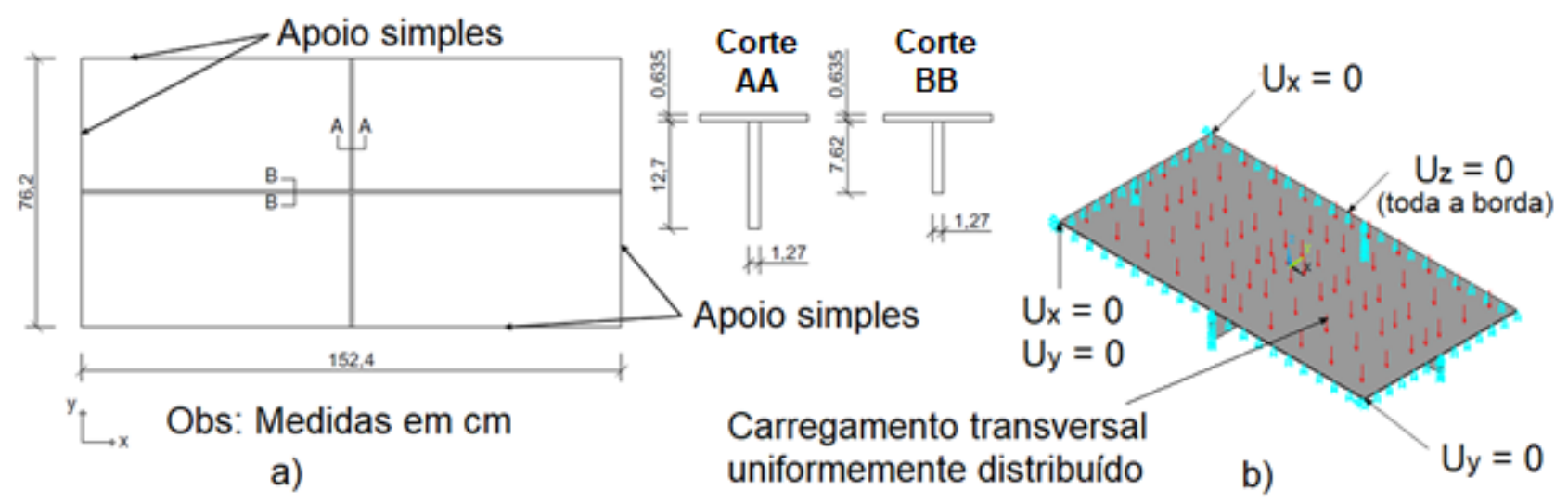

Figura 7. Placa retangular com dois enrijecedores ortogonais: (a) modelo físico [Adaptada de Silva (2010)] e (b) condições de contorno e carregamento.

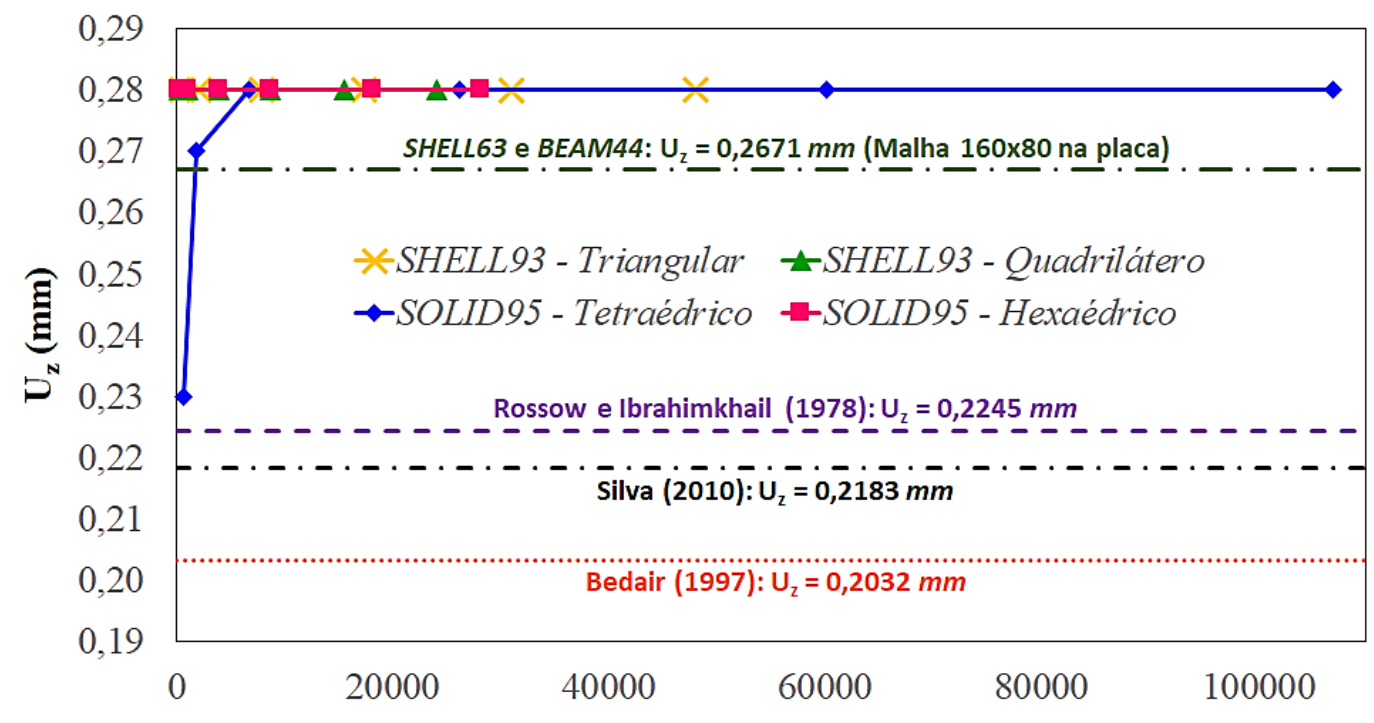

Número de elementos finitos

Figura 8. Resultados numéricos e análise de convergência de malha para placa retangular com dois enrijecedores ortogonais.

Assim como no caso anterior, é possível observar através da Fig. 8 que existe uma convergência entre os resultados gerados pelos 4 modelos computacionais propostos. 
$\mathrm{Na}$ Tabela 2, as deflexões no centro da placa obtidas com os modelos propostos e suas respectivas malhas independentes são comparadas com os resultados publicados por Rossow e lbrahimkhail (1978), Bedair (1997) e Silva (2010).

Tabela 2. Resultados para placa retangular com dois enrijecedores ortogonais.

\begin{tabular}{|c|c|c|c|c|c|c|c|}
\hline \multirow{2}{*}{$\begin{array}{c}\text { Placa } \\
\text { Enrijecida }\end{array}$} & \multicolumn{2}{|c|}{ SHELL 93} & \multicolumn{2}{|c|}{ SOLID 95} & \multirow{2}{*}{$\begin{array}{c}\text { Rossow e } \\
\text { Ibrahimkhail } \\
\text { (1978) }\end{array}$} & \multirow{2}{*}{$\begin{array}{l}\text { Bedair } \\
(1997)\end{array}$} & \multirow{2}{*}{$\begin{array}{c}\text { Silva } \\
(2010)\end{array}$} \\
\hline & Triangular & Quadrilátero & Tetraédrico & Hexaédrico & & & \\
\hline Malha & $\begin{array}{l}\text { M2: } 2.040 \\
\text { elementos }\end{array}$ & $\begin{array}{l}\text { M2: } 1.020 \\
\text { elementos }\end{array}$ & $\begin{array}{c}\text { M4: } 26.166 \\
\text { elementos }\end{array}$ & $\begin{array}{l}\text { M3: } 3.928 \\
\text { elementos }\end{array}$ & & & \\
\hline$U_{z}(\mathrm{~mm})$ & 0,2779 & 0,2780 & 0,2782 & 0,2781 & 0,2245 & 0,2032 & 0,2183 \\
\hline
\end{tabular}

Observa-se, novamente, que os 4 modelos desenvolvidos no presente estudo apresentam boa concordância entre si, porém, nesse caso, divergem em torno de 19\% a $27 \%$ dos resultados das referências utilizadas para a verificação.

Como já mencionado na introdução, Silva (2010) utilizou o MEF para solução do problema, empregando o elemento SHELL63 para a modelagem da placa e o elemento BEAM44 para a modelagem dos enrijecedores. O elemento SHELL63 possui 6 graus de liberdade por nó (assim como o elemento SHELL93 utilizado nesta verificação), porém possui apenas 4 nós e funções de interpolação lineares que the conferem menor acurácia que o SHELL93. Além disso, uma malha pouco refinada (40x20 na placa) com 860 elementos no total foi usada em Silva (2010), podendo esses fatores terem sido os responsáveis pela diferença de resultados encontrada.

Por esse motivo, esse exemplo numérico foi novamente simulado reproduzindo o modelo empregado por Silva (2010), mas com uma malha mais refinada (160x80 na placa), o que totalizou 13.040 elementos na estrutura como um todo (placa e enrijecedores). $O$ resultado obtido com essa simulação está também mostrado no gráfico da Fig. 8, onde é possível notar uma maior proximidade do resultado dessa nova simulação com os resultados obtidos pelos modelos propostos no presente trabalho.

É importante salientar que, dentre todos os modelos computacionais considerados na Fig. 8 e na Tabela 2, os que possuem menos simplificações teóricas são os que utilizam o elemento finito tridimensional SOLID95, por isso os valores com maior acurácia são os obtidos pelos modelos que empregam esse tipo de elemento. 
A Figura 9 mostra a configuração deformada da placa enrijecida com dois enrijecedores ortogonais, com a indicação no centro da placa das deflexões transversais obtidas com as respectivas malhas independentes de cada modelo.

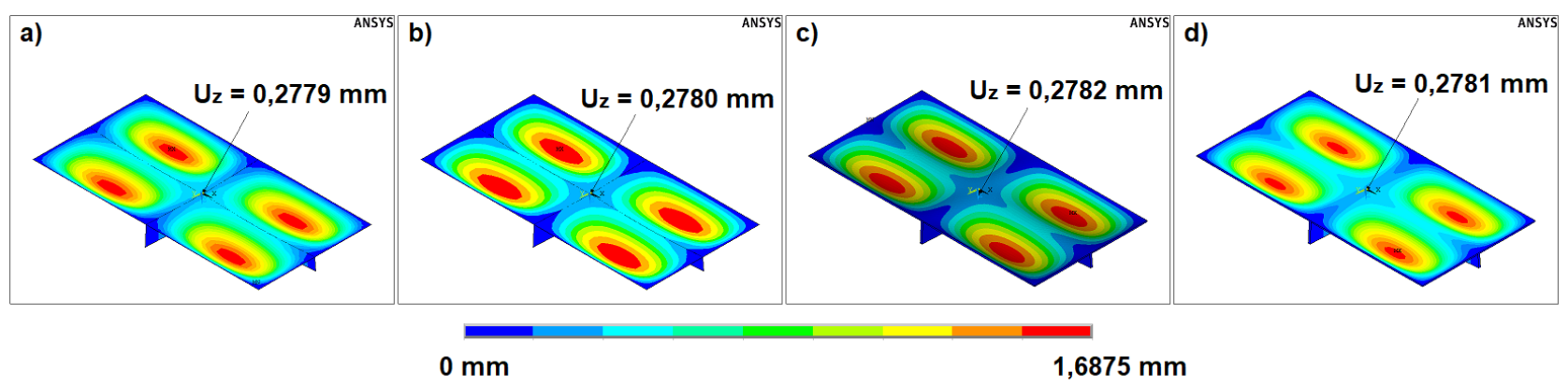

Figura 9. Deformada da placa retangular com dois enrijecedores ortogonais: a) SHELL93 - Triangular, b) SHELL93 - Quadrilátero, c) SOLID95 - Tetraédrico e d) SOLID95 - Hexaédrico.

O comportamento das deflexões da placa com dois enrijecedores ortogonais apresenta certa coerência física, apresentando quatro regiões em vermelho onde acontecem as deflexões máximas em cada um dos quatro quadrantes da placa. Essas regiões podem ser vistas na Fig. 9 para os 4 modelos computacionais desenvolvidos, os quais, diante do exposto, podem ser considerados devidamente verificados.

\section{CONSIDERACOES FINAIS}

Observa-se que os 4 modelos computacionais desenvolvidos apresentam resultados que convergem entre si para todas as simulações realizadas. Nota-se que o elemento SOLID95 na sua versão tetraédrica conduz a resultados com diferenças bastante elevadas quando utilizadas malhas grosseiras, ou seja, malhas com poucos elementos. As modelagens a partir dos elementos tipo SHELL93 são as que mais rápido convergem para os resultados finais de deflexão central das placas e, por possuírem malhas com menor quantidade de nós e elementos, apresentam, portanto, melhor eficiência computacional. Conclui-se que as diferentes modelagens computacionais realizadas apresentam resultados compatíveis com as referências científicas publicadas e, por isso, comprova-se que a metodologia utilizada neste estudo se mostra útil para obtenção de resultados de deflexão transversal em placas enrijecidas submetidas a esforços 
transversais uniformemente distribuídos. Em trabalhos futuros, pretende-se utilizar os modelos numéricos desenvolvidos e verificados neste trabalho para estudos de otimização geométrica de placas com enrijecedores.

\section{AGRADECIMENTOS}

Os autores agradecem ao CNPq, CAPES e FAPERGS pelo apoio financeiro.

\section{REFERENCIAS}

Ansys Inc. ANSYS User's Manual: Analysis Systems, 2009.

Bathe, Klaus-Jürgen. Finite element procedures. Englewood Cliffs: Prentice-Hall, 1996.

Bedair, Osama K. (1997). Analysis of stiffened plates under lateral loading using sequential quadratic programming (SQP). Computers \& Structures Vol. 62, n. 1, fev. 1997, p. 63-80.

Marinho, Ivy J.P. Projeto ótimo de estruturas metálicas de arquibancadas reutilizáveis via ANSYS, Dissertação (Mestrado) - PUC-Rio, 2002.

Rossow, Mark P. e Ibrahimkhail, A. K. Constraint Method Analysis of Stiffened Plates. Computers \& Structures, Vol. 8, fev. 1978, p. 51-60.

Salomon, Ami. An Evaluation of Finite Element Models of Stiffened Plates. Dissertação (Mestrado) - Massachusetts Institute of Technology, 2000.

Sapountzakis, Evangelos J. e Katsikadelis, John T. Analysis of Plates Reinforced with Beams. Computational Mechanics, Vol. 26, Jul. 2000, p 66-74.

Silva, Hugo B.S. Análise numérica da influência da excentricidade na ligação placaviga em pavimentos usuais de edifícios. Dissertação (Mestrado) - Universidade Federal de São Carlos, 2010.

Szilard, Rudolph, Theories and applications of plate analysis: Classical Numerical and Engineering Methods. Hoboken, New Jersey: John Wiley \& Sons, 2004.

Zienkiewicz, Olgierd. The finite Element Method in Engineering Science. $2^{\mathrm{a}}$ ed. London: McGraw- Hill, 1971. 